www.jmscr.igmpublication.org

Impact Factor (SJIF): 6.379

Index Copernicus Value: 79.54

ISSN (e)-2347-176x ISSN (p) 2455-0450

crossrefDOI: https://dx.doi.org/10.18535/jmscr/v6i10.189

\title{
Fractured Intravenous Cannula as Foreign Body
}

Authors

\section{Dr Dinesh Singh Chauhan, Dr Navneet Jain}

GDMC Dehradun, India

\begin{abstract}
Intravascular placement of plastic catheters is associated with a number of complications. Fracture of these intravascular catheters is not uncommon, however these cases are uder reported. We introduce the fracture of peripheral intravenous cannula while its removal from a superficial vein in right cubital fossa. The broken piece of the intravenous cannula was identified using ultrasound. Under sterile conditions, the broken piece of the cannula was removed from the vein under local anaesthesia.
\end{abstract}

\section{Case Report}

A 21 year old primigravida was admitted at term for normal vaginal delivery, labour was induced but due to foetal distress emergency LSCS was performed. Parentral drugs and fluids were given for three days via the intravenous cannula in the right cubital fossa. During removal of the intravenous cannula it fractured completely at the site of entry into the skin and the broke part could be felt on palpation in the right cubital fossa. Ultrasound of the right cubital fossa revealed a linear echogenic foreign body in superficial vein of right cubital fossa. The patient was taken to OT for removal of the foreign body under local anaesthesia. The right forearm was cleaned and draped with sterile sheets and local anaesthesia was infiltrated in the line of proposed skin incision. A transverse skin incison was made in the cubital fossa and the cannula was removed using artery forceps Figure 1. Haemostasis was achieved by pressure. The skin incision was closed with 3-0 ethilon and aseptic dressing was applied.

\section{Discussion}

Peripheral venous cannulation is the commonest vascular surgical procedure for almost every patient who arrives in labor. In modern medical practice, up to $80 \%$ of hospitalised patients receive intravenous therapy at some point during their admission ${ }^{[1,2]}$. Peripheral venous cannulation is associated with complications such as infiltration, thrombophlebitis, venous spasm, hematoma, air embolism, catheter associated blood stream infection, and also injury to tendon and nerve $\mathrm{e}^{[3]}$. Catheter fracture is a known complication of intravascular placement of plastic catheters; the first report we identified was by Turner et $\mathrm{al}^{[4]}$ in 1954. Significant complications with an embolic catheter fragment include sepsis, endocarditis, cardiac perforation, air embolism, pneumothorax, myocardial infarction and atrial or 
ventricular $\operatorname{arrhythmias}^{[5]}$. The reasons for spontaneous fracture and migration of intravenous cannula would be repeated failed attempts of insertion, inadequate expertise, loss of structural integrity due to repeated attempts with the same cannula or inferior quality of intravenous cannula or prolonged peripheral intravenous cannulation which is contrary to protocols for intravenous access $^{[6]}$. Current guidelines from the United Kingdom $^{[7]}$ and Australia ${ }^{[8]}$ recommend routine replacement of peripheral intravenous catheters every 48-72 hours to prevent painful infusion phlebitis and rare but life threatening peripheral catheter related bacteraemia. The findings suggested that the probable cause of the catheter fracture was partial transection from either trying to reinsert the needle into the already advanced catheter or advancing both catheter and needle with the needle partially withdrawn. This may have been further complicated by the anatomic curve in the vein at the antecubital fossa. In either case, attempts to remove the catheter likely completed the transection, leaving the intravascular distal fragment.

\section{Conclusion}

The process of cannulation can be divided into 4 steps; explanation and consent, preparation, procedure and aftercare. It is essential that health care providers who insert peripheral IV lines be familiar with the potential risk of catheter fracture and the proper emergency interventions required once it occurs and should document in the patients chart the date, time and reason for catheter removal and the integrity of the catheter as inspected.

\section{Bibliography}

1. Tjon JA, Ansani NT. Transdermal nitroglycerin for the prevention of intravenous infusion failure due to phlebitis and extra vasation. Ann Pharmacother 2000;34:1189-92,2.

2. Tager IB, Ginsberg MB, Ellis SE, et al. An epidemiologic study of the risks associated with peripheral intravenous catheters. Am J Epidemiol 1983;118:839-51.

3. Steele J. Practical IV Therapy. 2nd edition. Springhouse, PA: Springhouse Corp;1996: 68-69, 86-99.

4. Turner DD, Sommers SC. Accidental passage of a polyethylene catheter from cubital vein to right atrium: report of a fatal case. N Engl J Med 1954;251:744-5.

5. Surov A, Wienke A, Carter JM. Intra vascular embolization of venous catheter causes, clinical signs, and management: A systematic review. J Parenter Enteral Nutr. 2009;33:677-85.

6. Khadim MF, Leonard D, Moorehead RA, Hill C. Back to basics: iatrogenic intravenous cannula embolus. Ann R Coll Surg Engl. 2013;95(7):110-1.

7. British Medical Association: Healthcare associated infections: a guide for healthcare professionals. 2006.

8. Australian Department of Health and Ageing. Infection control guidelines for the prevention of transmission of infectious diseases in the health care setting.2004. 